\title{
Comparison of the Distributions of Ipsilaterally and Contralaterally Projecting Corticocortical Neurons in Cat Visual Cortex Using Two Fluorescent Tracers ${ }^{1}$
}

\author{
MARK A. SEGRAVES ${ }^{2}{ }^{2}$ AND GIORGIO M. INNOCENTI* \\ *Institute of Anatomy, University of Lausanne, Rue du Bugnon 9, 1011 Lausanne, Switzerland and $\ddagger$ Laboratory of Sensorimotor \\ Research, National Eye Institute, National Institutes of Health, Bethesda, Maryland 20205
}

\begin{abstract}
Using the retrograde fluorescent tracers Fast Blue and Diamidino Yellow we have studied the callosal and ipsilateral corticocortical connections between the cat's area $17 / 18$ border region and the posteromedial lateral suprasylvian visual area (PMLS), as well as the callosal connections of each of these regions with its contralateral homologue. The main goal was to determine whether single cortical neurons project with branching axons to more than one cortical target. In addition, the double-labeling technique enabled us to examine, within a single section of cortical tissue, the relative distributions of neurons with different cortical targets. Most corticocortical neurons labeled in the area 17/18 border region and in area PMLS projected to only one of the cortical injection sites tested. When two callosal neuron types were labeled in the same area, no double-labeled neurons were found. When ipsilateral corticocortical and callosal neurons were labeled in combination, a few double-labeled neurons were found in both cortical regions examined. The most common type of double-labeled neuron was located in area PMLS and projected bilaterally to the area $17 / 18$ border region. Our findings regarding the laminar distributions of ipsi- and contralaterally projecting neurons are in agreement with previous studies. In addition, we have found that, for callosal neurons within the upper layers of areas 17 and 18, neurons projecting to the contralateral area $17 / 18$ border are located in the lower half of layer II/III and in upper layer IV, whereas neurons projecting to contralateral area PMLS are restricted to the lower portion of layer II/III. In addition, for callosal neurons within the deep layers of area PMLS, neurons projecting to contralateral area PMLS are located throughout layers $V$ and $V I$, whereas neurons projecting to the contralateral area $17 / 18$ border are restricted to layer VI. There are numerous other possible targets for axon collaterals not examined in this paper. However, the scarcity of
\end{abstract}

Received October 5, 1984; Revised February 25, 1985;

Accepted February 27, 1985

\footnotetext{
1 This work was supported by Swiss National Science Foundation Awards 88.959.0.81, 3.628.0.80, and 3.922.0.83. We gratefully acknowledge the technical assistance of Marie Christine Cruz, preparation of figures by $M$. Gaillard, and secretarial help of Jean Steinberg. We thank Drs. Michael E. Goldberg and Alan C. Rosenquist for their comments on the manuscript, and Dr. Stephanie Clarke for assistance with the control injections in mice.

${ }^{2}$ To whom correspondence should be addressed, at Laboratory of Sen sorimotor Research, National Eye Institute, National Institutes of Health, Building 10, Room 10B09, Bethesda, MD 20205.
}

neurons with multiple projections demonstrated in this study reflects the high degree of specificity of cortical connectivity. This anatomical organization may be the basis for a precise channeling of differential information at the single neuron level.

Each of the cat's numerous cortical representations of the visual hemifield is interconnected with many other cortical areas in both hemispheres. Two strongly interconnected regions which have been the subject of intensive anatomical, physiological, and behavioral studies are the area $17 / 18$ border region and the posteromedial lateral suprasylvian area (area PMLS) of Palmer et al. (1978). The existence of extensive interconnections contrasts with the many differences between these two areas. Areas 17 and 18 receive their primary thalamic input from the lateral geniculate nucleus (LGN). The main subcortical input of area PMLS is from the striate recipient zone of the lateral posterior complex (Raczkowski and Rosenquist, 1983). The receptive field properties of neurons in these areas reflect these differences in thalamic inputs. In contrast to the small, finely tuned receptive fields of areas 17 and 18, area PMLS receptive fields are relatively large and unselective for stimulus orientation, and most respond poorly to stationary stimuli (Spear and Baumann, 1975; Camarda and Rizzolatti, 1976). In addition, these regions apparently make very different contributions to visual processing. One very important function of areas 17 and 18 appears to be the fine analysis of visual detail. Lesions of areas 17 and 18 in cats result in a reduction in visual acuity (Berkley and Sprague, 1979). Area PMLS appears to be part of a group of extrastriate visual areas important for form discrimination and the interhemispheric transfer of visual learning (Sprague et al., 1977; Berlucchi et al., 1979), or may even contribute to the cortical control of eye movements (Spear et al., 1983)

In this study, we have used the fluorescent tracer techniques developed by Kuypers and colleagues (Kuypers et al., 1977; Bentivoglio et al., 1980; Keizer et al., 1983) to study the connections between the cat's area $17 / 18$ border region and area PMLS. It is known that the area $17 / 18$ border region and area PMLS are callosally connected with their homologous contralateral regions. In addition, strong callosal and ipsilateral connections exist between the area $17 / 18$ border region and area PMLS. It is also known from retrograde transport studies using horseradish peroxidase (HRP) (e.g., Gilbert and Kelly, 1975; Shatz, 1977; Keller and Innocenti 1981: Segraves and Rosenquist, 1982b; Symonds and Rosenquist, 1984 b) that corticocortically projecting neurons can have a specific laminar distribution depending upon the area to which they project. For example, in area PMLS, layer VI is the primary origin for ipsilateral and contralateral projections to the area $17 / 18$ border region, whereas layer III is the main source of projections to contralateral PMLS. In other cases, however, the laminar distribution of neurons 
contributing to different pathways seems identical or, at least, very similar. For example, at the cat area $17 / 18$ border, layer III contains neurons which project ipsilaterally to area PMLS, as well as neurons projecting contralaterally to the area $17 / 18$ border region or to area PMLS. In light of the existing data for interconnections between the area $17 / 18$ border region and area PMLS, as well as what is known about their distinct contributions to visual processing and behavior, we wanted to determine whether the individual neurons which form these interconnections project to more than one termination site in either ipsilateral or contralateral cortex, and to compare within single brain sections the laminar distributions of neurons projecting to different termination sites. Our basic finding is that each of several cortical projections arising from a single visual area originates from a separate and distinct population of neurons, although different populations are frequently intermixed within a particular cortical layer. In addition, this work reveals several subtleties of the laminar distribution patterns that were not apparent in single-label studies. A preliminary report of this study was presented elsewhere (Segraves and Innocenti, 1982).

\section{Materials and Methods}

Experimental design. Three classes of projections interconnecting the area $17 / 18$ border region and area PMLS were examined using the fluorescent tracer techniques developed by Kuypers and colleagues (Kuypers et al., 1977; Bentivoglio et al., 1980; Keizer et al., 1983). These classes were: (1) homotopic callosal projections-connecting homologous areas in opposite hemisphercs, for example, the left arca PMLS with the right arca PMLS; (2) heterotopic callosal projections-connecting heterologous areas in opposite hemispheres, for example, the left area PMLS with the right area 17/ 18 border; and (3) insilateral corlicocortical projections belween the area 17/ 18 border region and area PMLS within the same hemisphere. Given the three classes of projections examined, neurons either in the area 17/18 border region or in area PMLS could be tested for their projection to each of the three possible pairs of targets using four combinations of injections illustrated in Figure 1.

Techniques. Data from seven cats are included in this report. Each cat was anesthetized with sodium pentobarbital $(40 \mathrm{mg} / \mathrm{kg}$ ) and then given 0.2 $\mathrm{mg}$ of atropine sulfate subcutaneously. Two skull openings were made exposing the intended injection sites. One mililiter of $2 \%$ lidocaine was injected into the skin and muscle was cut to expose the skull. One milliliter of penicillin was injected intramuscularly

In an effort to optimize the possibilities for double labeling of neurons with branching axons, we attempted to include large and equivalent portions of the visual field representation within the two cortical injection sites. Typically, five injections at $1-\mathrm{mm}$ intervals rostrocaudally were made in each of the two regions of interest. Injections in the area $17 / 18$ border region were meant to include the vertical meridian and area centralis representations, and were located along the crown of the lateral gyrus between stereotaxic levels posterior 4.0 and anterior 2.0. Injections in area PMLS were meant to include the vertical meridian and area centralis representations of PMLS and involved as much as possible of the medial bank and fundus of the middle suprasylvian sulcus between stereotaxic levels posterior 3.0 and anterior 2.0.

The flourescent tracers Fast Blue and Diamidino Yellow dihydrochloride were used. Both tracers are transported retrogradely from axon terminals to neuron soma. These two dyes have the additional advantage that they both fluoresce in response to similar excitation wavelengths in the ultraviolet range, enabling them to be viewed simultaneously without changing filters. Fast Blue-labeled neurons have a blue fluorescent cytoplasm and occasionally a silvery nucleus. Diamidino Yellow-labeled neurons contain a bright yellowishwhite nucleus and occasionally a very pale yellow cytoplasm. Neurons labeled by both Fast Blue and Diamidino Yellow have a yellow nucleus surrounded by pale blue cytoplasm. Together, these characteristics enable one to easily distinguish neurons labeled by Fast Blue or Diamidino Yellow, as well as to identify neurons labeled by both tracers. Two forms of Fast Blue were used: dye number 253/152, a water soluble form, and dye number $253 / 50$. The total amount injected ranged from 20 to $75 \mu \mathrm{g}$. The procedure we preferred was to inject approximately $20 \mu \mathrm{g}$ of Fast Blue 253/50 in a series of five $0.25-\mu$ injections of a $1.5 \%$ solution. The water-soluble form of Fast Blue was found to be unsatisfactory because of its tendency to diffuse out of labeled neurons, causing artifactual labeling of adjacent neurons and glia. Diamidino Yellow dihydrochloride 288/26 produces fluorescent labeling similar to that of Nuclear Yellow but has the advantage that it does not leak out of labeled neurons and can be used with survival times comparable to those required for Fast Blue labeling (Keizer et al., 1983). Diamidino Yellow was injected in a total amount of $50 \mu \mathrm{g}$. Typically, five $0.5-\mu$ injections of a $2.0 \%$ solution were made. Fast Blue and Diamidino Yellow were injected with separate Hamilton syringes to avoid contamination.

Survival times ranged from 6 to 14 days with 6 days being the most common. At the end of the survival period, the cat was anesthetized deeply with sodium pentobarbital and perfused first with saline, followed by 2 liters of $30 \%$ formalin in $0.1 \mathrm{M}$ cacodylate or phosphate buffer $(\mathrm{pH} 7.2)$, and finally with 2 liters of $20 \%$ sucrose in the same buffer. The brain was removed from the skull and left overnight in a $30 \%$ buffered sucrose solution. Within $48 \mathrm{hr}$ of the time of sacrifice, the brain was sectioned in a cryostat. One $30-\mu \mathrm{m}$ section every $150 \mu \mathrm{m}$ was mounted on a gelatin-coated slide, air dried, and stored at $4^{\circ} \mathrm{C}$. At least every other section (i.e., one $30-\mu \mathrm{m}$ seclion $/ 300 \mu \mathrm{rl}$ ) through the region containing labeled neurons in areas 17, 18, and PMLS and in the LGN was examined with a Zeiss fluorescence microscope. Every section was examined in the vicinity of double-labeled neurons (i.e., one 30 $\mu \mathrm{m}$ section $/ 150 \mu \mathrm{m})$. We found that the combinations of excitation/barrier filters of 395 to $425 / 450$ or 402 to $408 / 435 \mathrm{~nm}$ were optimal for the differential visualization of Fast Blue- and Diamidino Yellow-labeled neurons.

As mentioned above, we attempted to position our injections at equivalent retinotopic regions within the two injection areas. To ensure that we were successful, the positions of Fast Blue- and Diamidino Yellow-labeled neurons in the LGN were examined in each experiment. The LGN C-laminae project to areas 17, 18, and PMLS (Rosenquist et al., 1974; LeVay and Gilbert, 1976), and each of these projections is retinotopically organized (Raczkowski and Rosenquist, 1980; Tong et al., 1982). Thus, the distributions of Fast Blue- and Diamidino Yellow-labeled neurons in the C-laminae provided a direct comparison of the relative size and retinotopic correspondence of the two injection sites. In addition, the distributions of Fast Blue- and Diamidino Yellow-labeled neurons within an area that we intended to study were compared to ensure that there was substantial overlap between the two neuron populations. This comparison was accomplished with the aid of a computer. For each of a series of brain sections, labeled neurons were projected onto a line, parallel to the cortical surface, running midway between white matter and cortical surface. A histogram was then constructed for each section showing the density distribution of labeled neurons along this line. Histograms from a series of adjacent sections were aligned along a rostrocaudal axis corresponding to the midpoint of the crown of the lateral gyrus for areas 17 and 18, and to the fundus of the middle suprasylvian sulcus for area PMLS. This provided maps of the distributions of labeled neurons in these two areas. A comparison of the aligned histograms for $\Gamma$ ast Blue- and Diamidino Yellow-labeled neurons in each animal revealed whether or not there was extensive overlap between the two neuron populations.

Selected sections from the regions of highest overlap of Fast Blue and Diamidino Yellow labeling were plotted using a fluorescence microscope with computer-driven $X-Y$ stage. The step size of the computer-driven $X-Y$ stage was $1 \mu \mathrm{m}$, thus allowing us to plot the distributions of Fast Blue- and Diamidino Yellow-labeled neurons within a single brain section with very high resolution.

To ensure that Fast Blue and Diamidino Yellow had the potential to yield a high percentage of double labeling, superimposed injections of the two dyes were made in the cortex of several mice. Within the region of most intense thalamic neuron labeling in these preliminary experiments, we found that $100 \%$ of the neurons were double labeled.

The borders between laminae werc dotcrmincd in Nissl stained sections using the criteria of Otsuka and Hassler (1962) for areas 17 and 18 and Sanides and Hoffman (1969) for area PMLS. The border between areas 17 and 18 was also determined from Nisst-stained seclions. The border of area PMLS has not been defined based upon architectonic criteria. In agreement with the electrophysiological map of Palmer et al. (1978), we defined area PMLS as the region of cortex located on the medial bank of the middle suprasylvian sulcus extending from the fundus to the medial lip of the middle suprasylvian sulcus and rostrocaudally from approximately anteroposterior level +9.0 to the junction of the middle and posterior suprasylvian sulci.

\section{Results}

Results from a total of seven cats are included in this report. Two cats reccived arca 17/18 border region and area PMLS injections in the same hemisphere. Bilateral area $17 / 18$ border or area PMLS injections were made in two pairs of cats. In one additional cat, the area $17 / 18$ border region and area PMLS were injected in opposite hemispheres. Our criteria for including an animal in this study were as follows. Our first criterion was that there be no labeling of LGN A-laminae by the area PMLS injection. Scattered neuronal labeling 


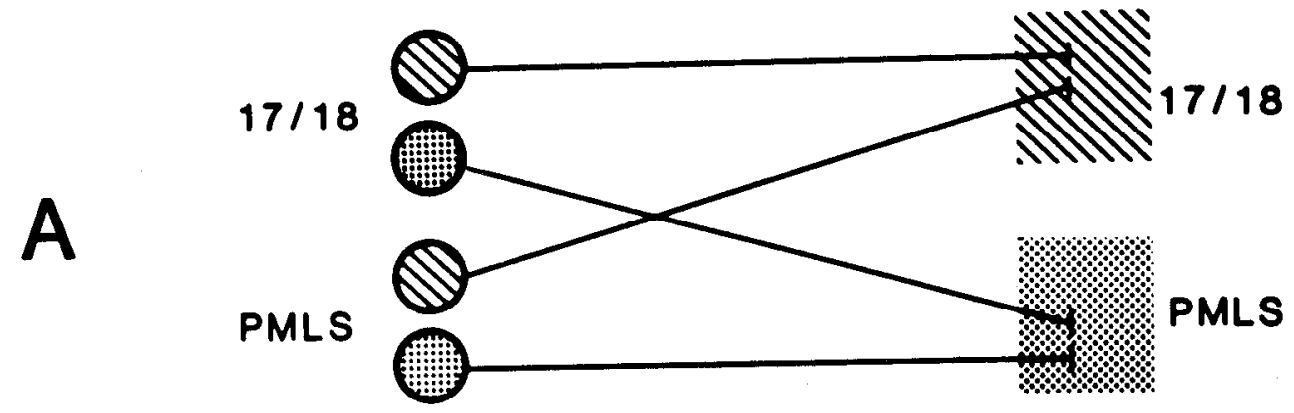

\section{$17 / 18$}

PMLS

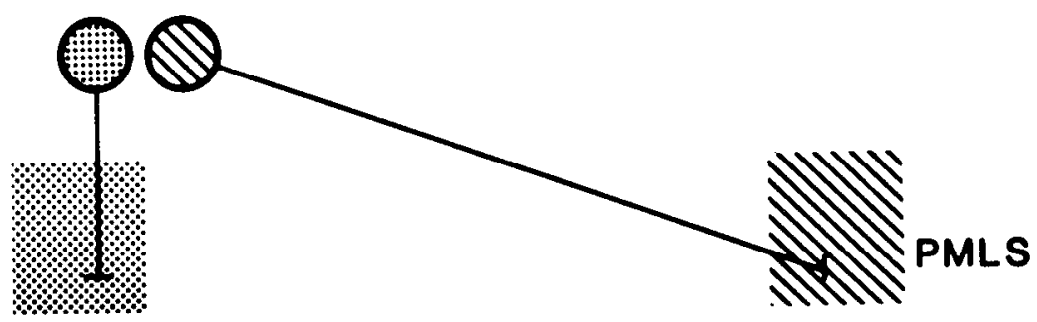

$17 / 18$

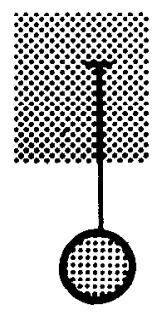

PMLS

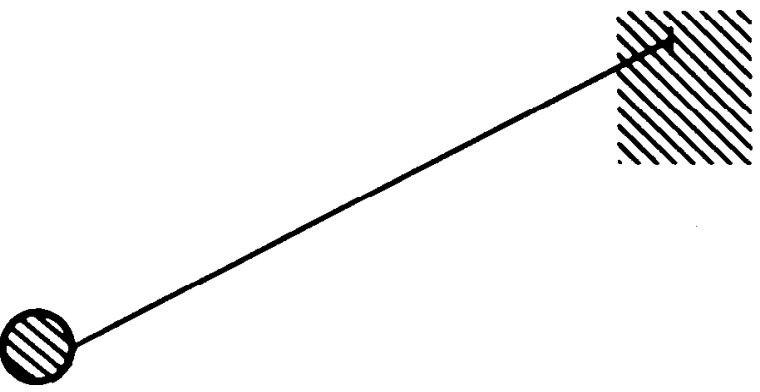

PMLS

$17 / 18$

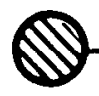

D

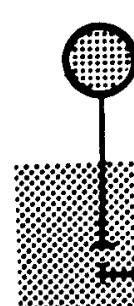

Figure 1. Four injection combinations of Diamidino Yellow and Fast Blue used to study the ipsilateral and callosal interconnections of the area $17 / 18$ border region, and area PMLS. Hatched squares and circles symbolize injection sites and labeled neurons. The left and right sides of this diagram denote opposite cerebral hemispheres. A, Right hemisphere injections of Diamidino Yellow in the area $17 / 18$ border region and Fast Blue in lateral suprasylvian area PMLS, labeling neurons in the left area $17 / 18$ border region and area PMLS with homotopic and heterotopic callosal projections. $B$, Bilateral injections of area PMLS, labeling neurons in the area $17 / 18$ border region with ipsilateral corticocortical and heterotopic callosal projections to area PMLS. C, Bilatera area $17 / 18$ border injections, labeling neurons in area PMLS with ipsilateral corticocortical and heterotopic callosal projections to the area $17 / 18$ border region. $D$, Left hemisphere Fast Blue injection in area PMLS combined with right hemisphere Diamidino Yellow injection in the area $17 / 18$ border region, labeling neurons in the left area $17 / 18$ border region and right area PMLS with ipsilateral corticocortical and homotopic callosal projections.

\section{$=$ FAST BLUE INJECTION}




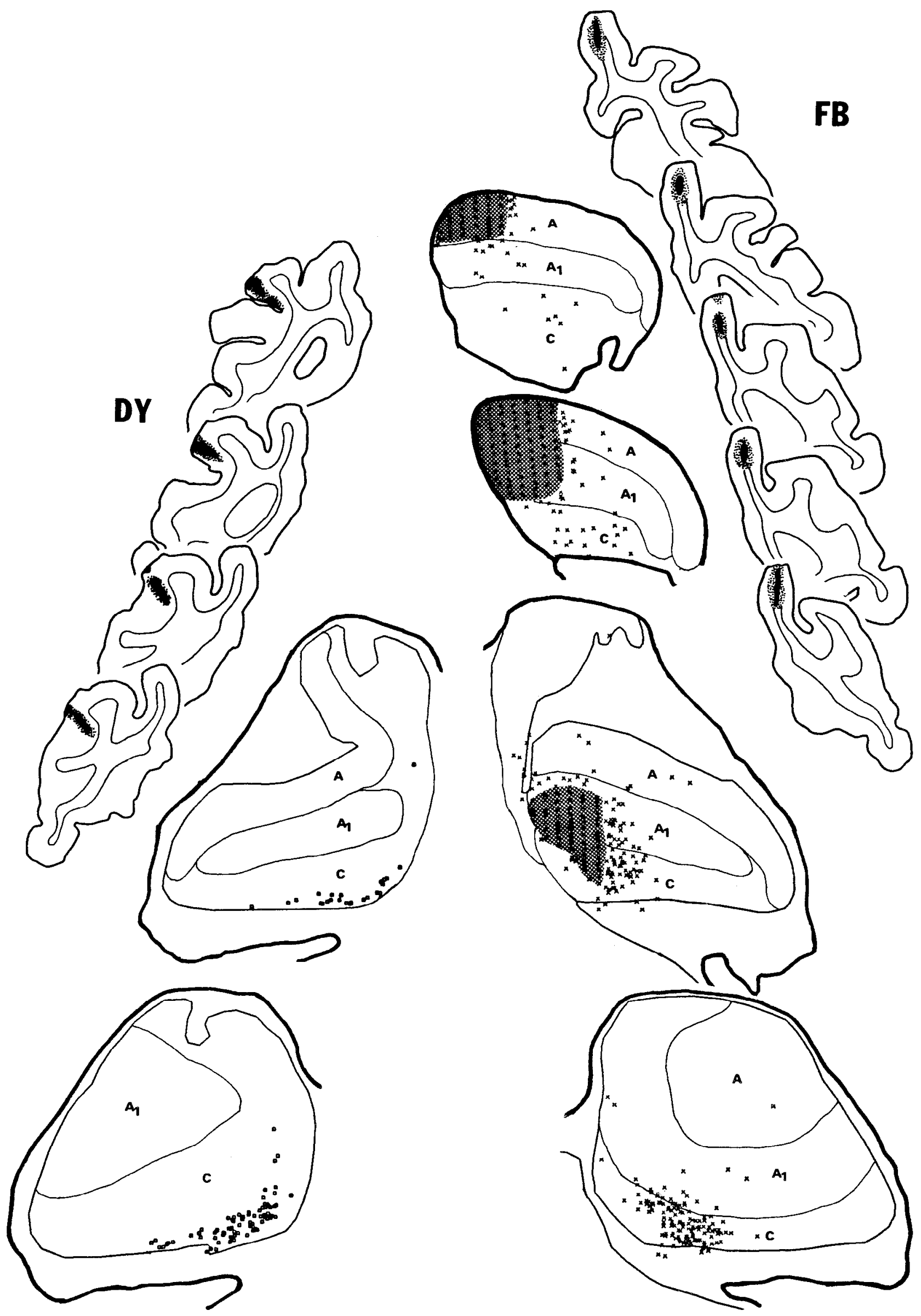

Figure 2. Cortical injection sites and LGN labeling following injections of Diamidino Yellow (DY) in lateral suprasylvian cortex of the left hemisphere and Fast Blue $(F B)$ in the crown of the lateral gyrus of the right hemisphere. Squares mark positions of Diamidino Yellow-labeled neurons in the left LGN. Crosses mark the positions of Fast Blue-labeled neurons in the right LGN. Shading in the right LGN marks regions of very dense Fast Blue labeling. Both the absence of A-laminae labeling resulting from the lateral suprasylvian injections and the similar distributions of C-laminae labeling in the two hemispheres apparent in this case were necessary criteria for inclusion of an animal in this study. 


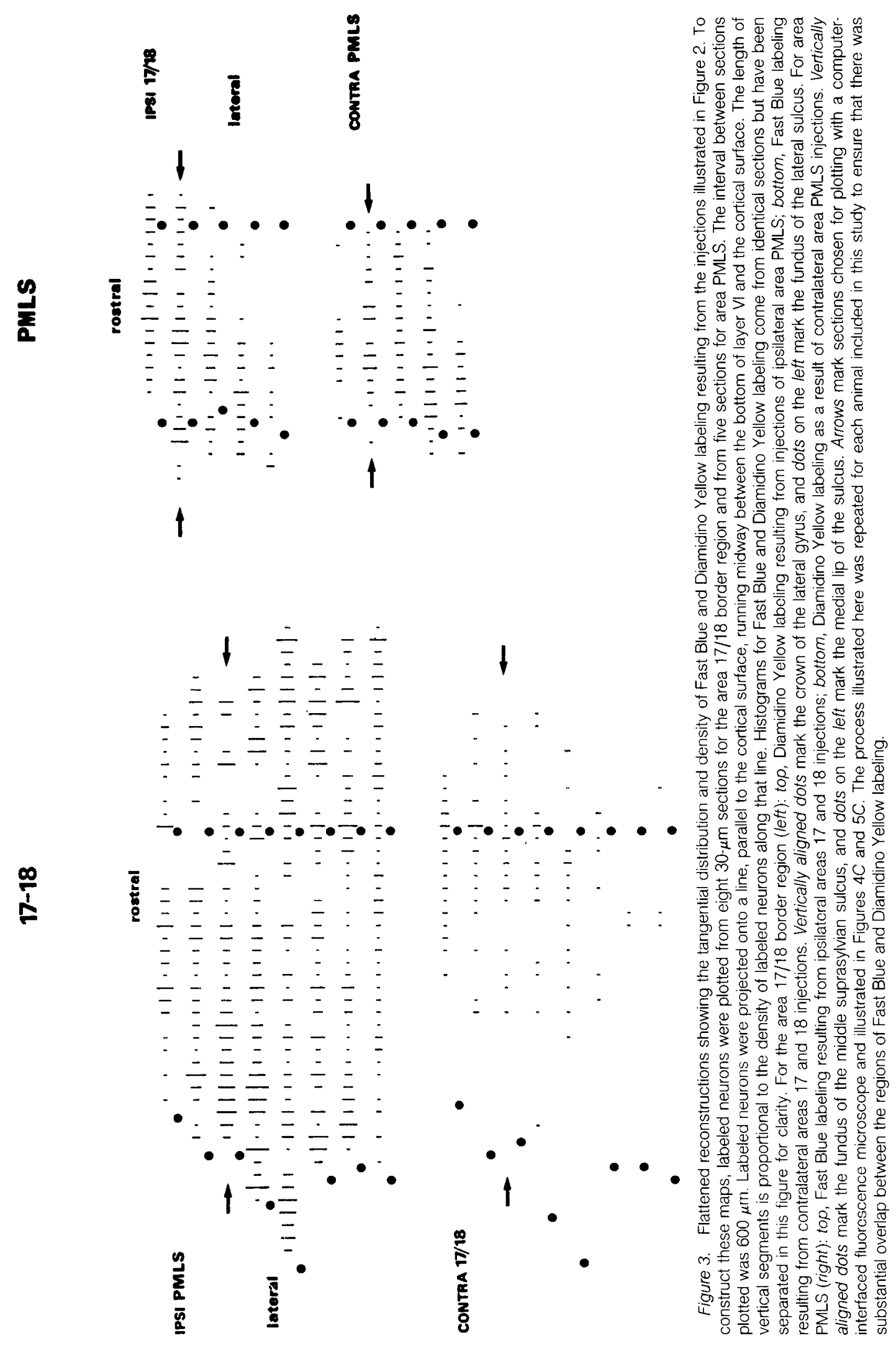




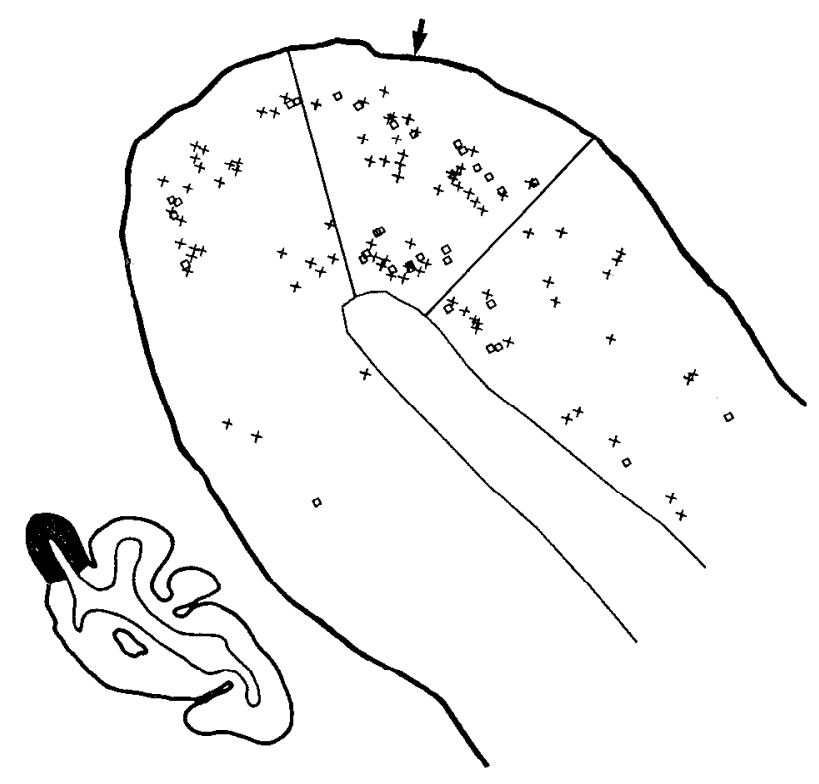

A
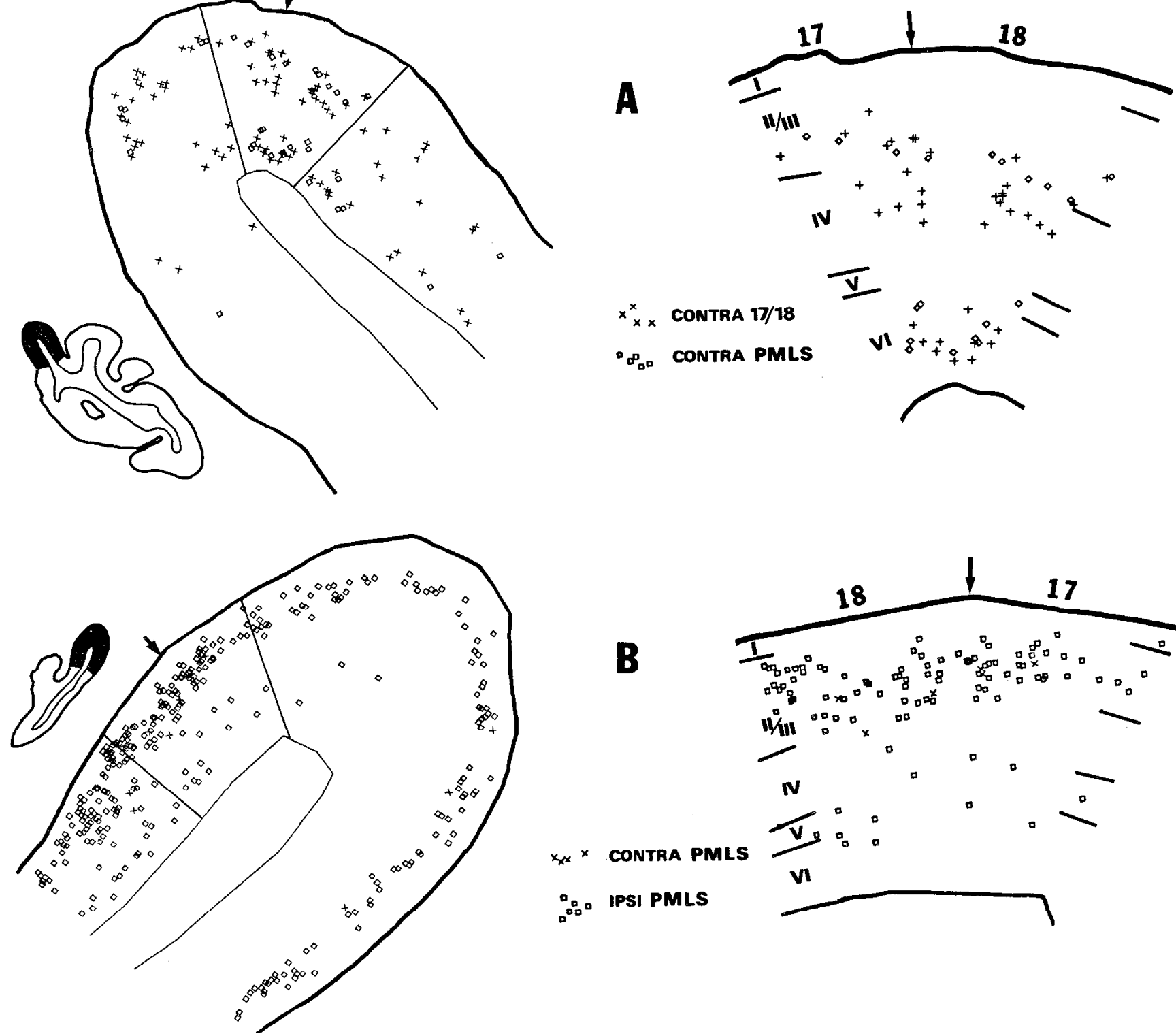

$x \times$ CONTRA PMLS

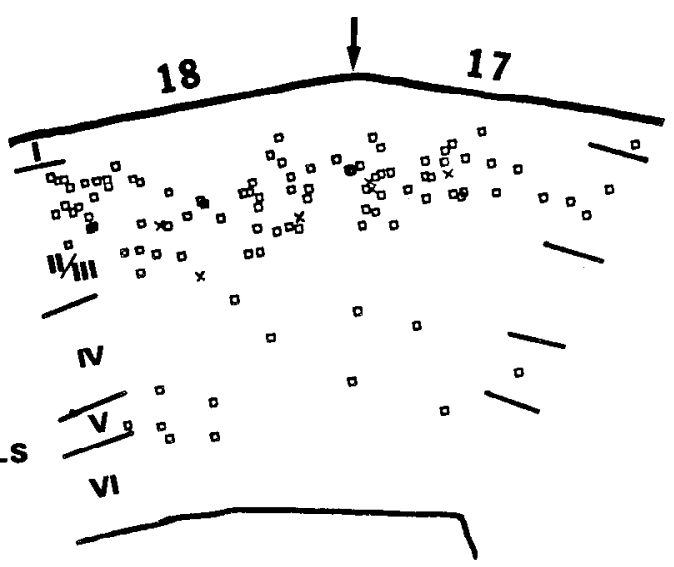

IPSI PMLS
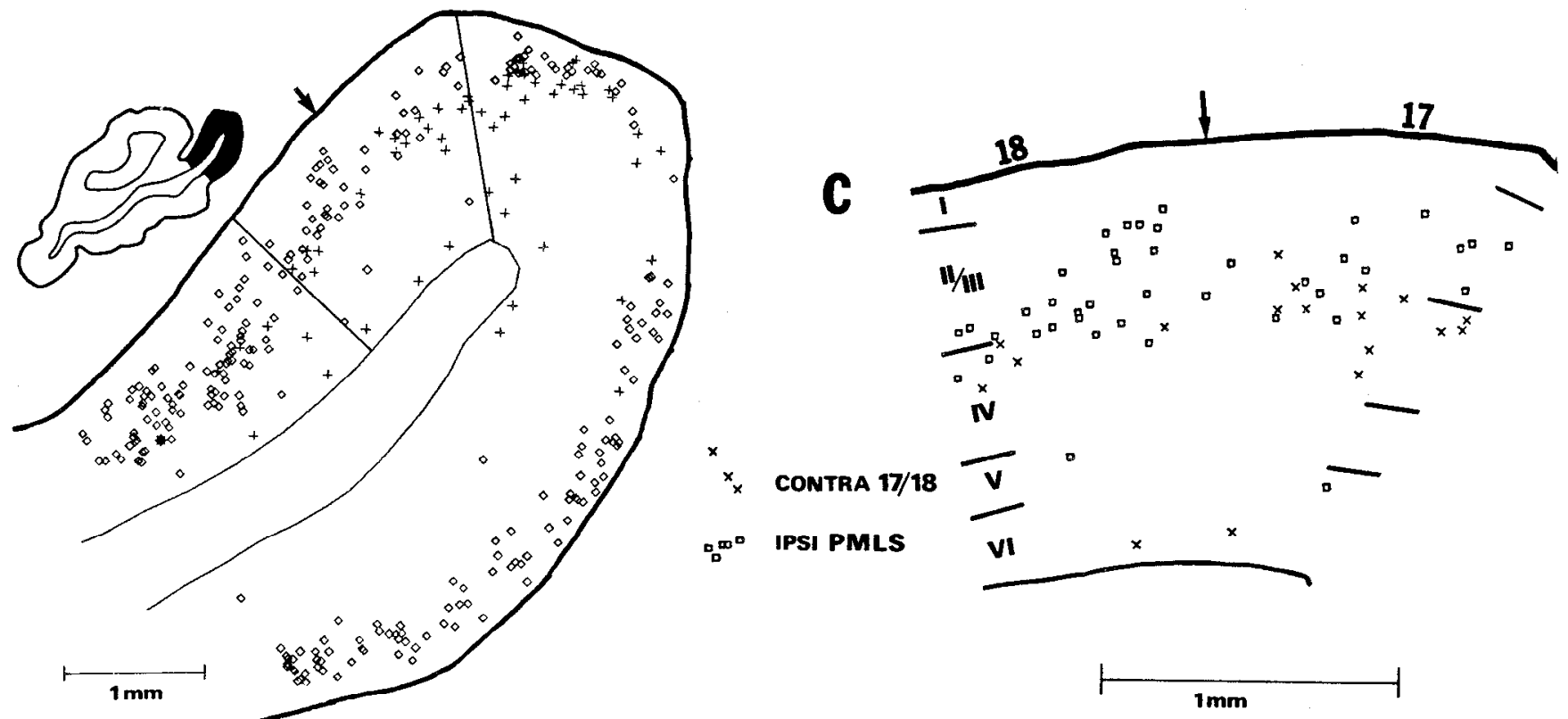

Figure 4. Labeling in areas 17 and 18. Arrows mark the area 17/18 border. The area 17/18 border region is enlarged on the right side of this figure. Crosses and squares mark the positions of Fast Blue- and Diamidino Yellow-labeled neurons, respectively, and their significance is indicated for each purliur of the figure; e.g., in A, crosses mark positions of neurons labeled by injections of contralateral areas 17 and 18, and squares mark positions of neurons labeled by injections of contralateral area PMLS. The asterisk in the left portion of $C$ marks the position of a single double-labeled neuron. 

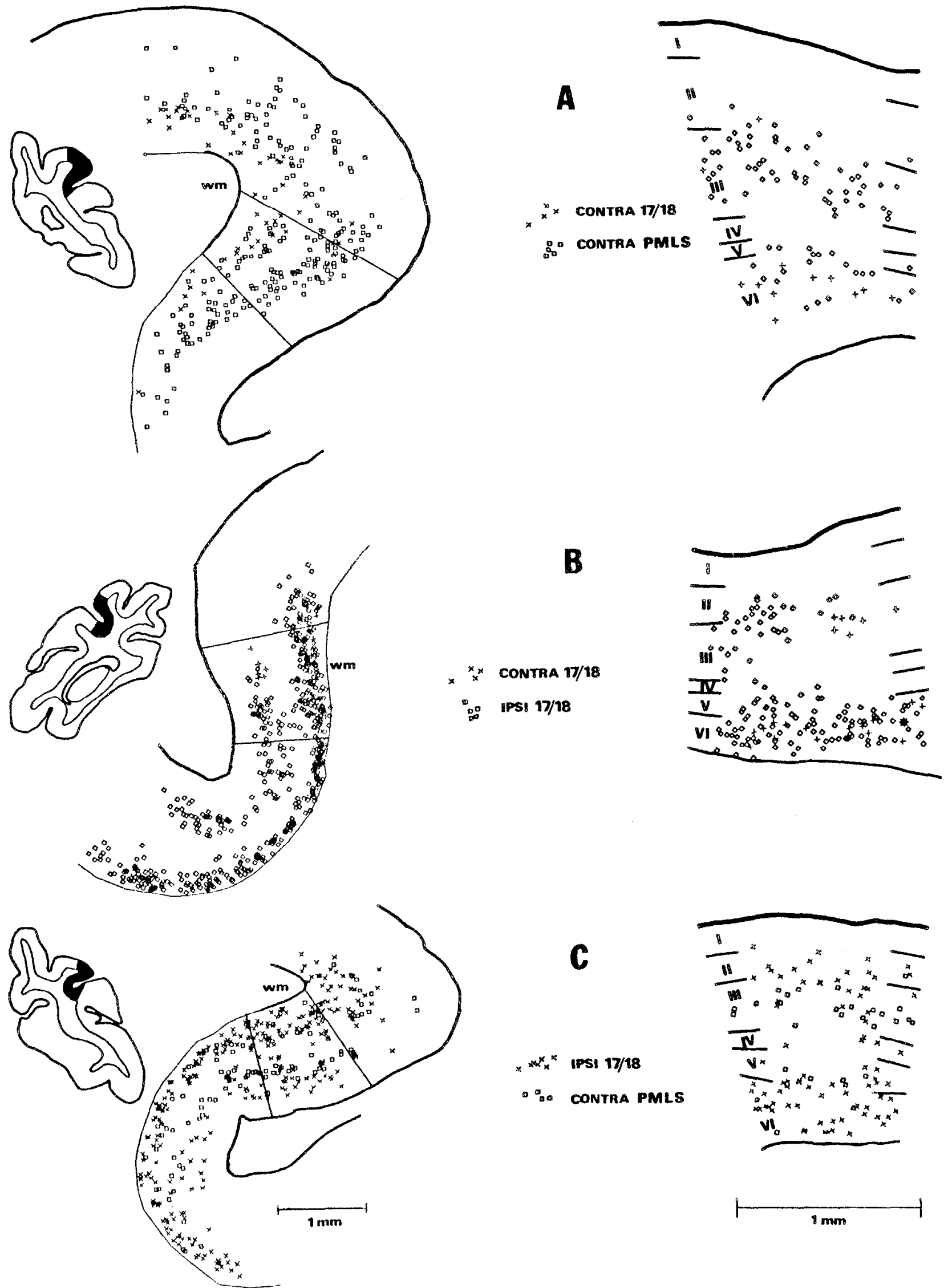

Figure 5. Labeling in the medial bank of the middle suprasylvian sulcus. At the anteroposterior levels depicted here, the approximate extent of area PMLS is from the fundus to the medial lip of the middle suprasylvian sulcus. The asterisk in the right portion of $B$ marks the position of a double-labeled neuron in layer Vl. wm, white matter. 
2114

Segraves and Innocent

Vol. 5, No. 8, Aug. 1985

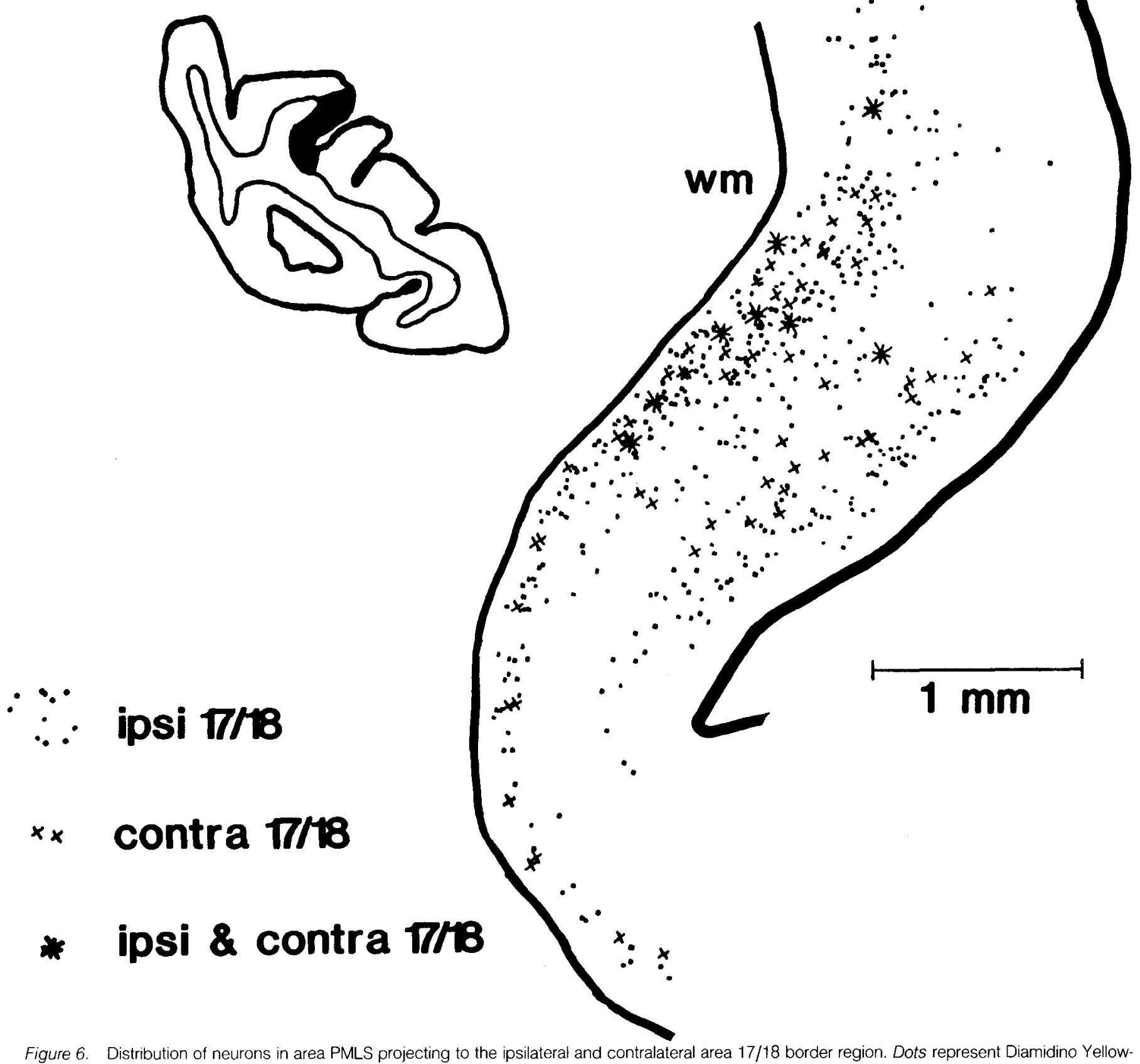

Figure 6. Distribution of neurons in area PMLS projecting to the ipsilateral and contralateral area $17 / 18$ border region. Dots represent Diamidino Yellow-
labeled neurons projecting ipsilaterally, crosses mark Fast Blue-labeled neurons projecting contralaterally, and asterisks mark double-labeled neurons experiments. $w m$, white matter.

within the LGN A-laminae occurred in several cats (excluded from this study) when dye spread from the fundus of the middle suprasylvan sulcus to underlying white matter. Fibers from the LGN run through the white matter located beneath the fundus of the middle
suprasylvian sulcus (Poliak, 1926; Shoumura, 1973), and diffusion into this region must have resulted in pickup of dye by LGN that this diffusion might also result in labeling of a descending projection from areas 17 and 18 and, thus, would confound our results. Our second criterion was that there be a close correspond once in the retinotopic distribution of LGN C-laminae labeling result- ing from both injection regions. Our third criterion was that there be extensive overlap of the regions of Fast Blue and Diamidino Yellow neuron labeling in the cortical area we wished to study. Figure 2 illustrates the injection sites and LGN label from a cat injected with Diamidino Yellow in its left area PMLS and Fast Blue in A-lamina labeling in the left LGN. In addition, there is a close correspondence in the position of C-lamina label between the two sides, indicating that the retinotopic positions of the injection areas overlapped. Figure 3 contains a series of stacked histograms from
adjacent sections comparing, for the left area $17 / 18$ border region, 
TABLE ।

Summary of the laminar distributions of corticocortical neurons with ipsilateral or contralateral projections in the area 17/18 border region and in area PMLS

Area 17/18 distributions are given in row 1 and those for area PMLS are given in row 2 . Enlarged boldface numerals indicate the primary laminae of origin for a particular projection. For example, the majority of neurons in the area 17/18 border region projecting to ipsilateral area PMLS are in layer I/III.

\begin{tabular}{ccccc} 
& IPSI $17 / 18$ & IPSI PMLS & CONTRA $17 / 18$ & CONTRA PMLS \\
\hline $17 / 18$ & & II /III,IV,V,VI & II /III,IV ,VI & II /III ,VI \\
\hline PMLS & II,III,IV,V,VI & & III, VI & II, III,V,VI \\
\hline
\end{tabular}

the distributions of neurons projecting to ipsilateral area PMLS and to the contralateral area $17 / 18$ border, and for the right area PMLS, the distributions of neurons projecting to the ipsilateral area 17/18 border region and to the contralateral area PMLS. This material was taken from the same animal illustrated in Figure 2. Note that there is a great deal of overlap between the two neuron populations in both instances. Arrows in Figure 3 indicate the positions of sections chosen for plotting with the computer-interfaced microscope and illustrated in Figures $4 C$ and $5 C$. The rostrocaudal extent of the region of overlap of the two labeled neuron populations ranged from 1800 to $9600 \mu \mathrm{m}$ in these experiments, with a mean rostrocaudal overlap of $4200 \mu \mathrm{m}$.

Neurons with homotopic and heterotopic callosal projections in areas 17 and 18. When neurons with homotopic callosal projections and neurons with heterotopic callosal projections were labeled in areas 17 and 18 (Fig. 4A), both neuron types were distributed in two distinct subzones (Innocenti, 1980): a superficial subzone consisting of layer $\|/ I\|$ and upper layer IV, and a deep subzone within layers $V$ and $V I$. The distributions of the two neuron types were closely overlapped, both tangentially and within laminae. A notable difference was that, in the superficial subzone, neurons with homotopic callosal projections were distributed within the bottom half of layer $I / I I I$ and the upper part of IV, whereas neurons with heterotopic callosal projections were restricted to the deep half of layer $\|/\|$ in this region. Although a direct comparison could only be made in the two cats receiving injections at the area $17 / 18$ border region and area PMLS in the same hemisphere, this pattern of distribution is also clear for neurons with heterotopic callosal projections in Figure $4 B$ and for neurons with homotopic callosal projections in Figure $4 C$. Within the deep subzone, most labeled neurons were located in layer $\mathrm{VI}$ with an occasional neuron found in layer $\mathrm{V}$. There was no apparent tangential segregation or clustering of these two callosal neuron types. Fast Blue- and Diamidino Yellow-labeled neurons were often found immediately adjacent to one another. However, no double-labeled neurons were found in areas 17 or 18 in either of the two animals in which areas 17, 18, and PMLS were injected in the same hemisphere. Without exception, each labeled neuron maintained only one of the two callosal projections examined in this experiment.

Neurons with ipsilateral corticocortical and heterotopic callosal projections in areas 17 and 18. Bilateral injections of area PMLS enabled us to compare, at the area $17 / 18$ border, the distributions of neurons with projections to ipsilateral arca PMLS to those with projections to contralateral area PMLS (Fig. 4B). As expected, neurons with ipsilateral projections were distributed throughout layer II/III, with a few additional neurons scattered in layers IV, V, and VI. Labeled neurons located below the layer III/IV border were primarily found in area 18. Heterotopic callosal ncurons, as described above (Fig. 4A), were primarily above the layer III/IV border. The two neuron types were intermixed in layer $\mathrm{I} / \mathrm{ll}$. No tangential segregation was evident within the region where ipsilaterally and contralaterally projecting neurons overlap. In the other cat receiving bilateral area PMLS injections, ipsilaterally projecting ncurons in the peripheral visual field representation of area 17 were grouped into clusters in some sections. However, this pattern of labeling was not present within the region where callosal neurons were labeled. Although none are illustrated in Figure $4 B$, one double-labeled neuron was found in area 18 of an adjacent section from this experiment. This single double-labeled neuron was the only one found within 19 sections that we examined in the region of good overlap between Fast Blue and Diamidino Yellow labeling. Each of the 19 sections examined had a density of labeled neurons similar to that of the section illustrated. A total of three double-labeled neurons were found within area 18 near the area $17 / 18$ border in the other cat receiving bilateral area PMLS injections. These three neurons were found within a total of 37 sections taken from both hemispheres and showing good overlap of Fast Blue and Diamidino Yellow labeling in the area $17 / 18$ border region.

Neurons with ipsilateral corticocortical and homotopic callosal projections in areas 17 and 18. Injections of ipsilateral area PMLS and the contralateral area $17 / 18$ border region enabled us to compare the distributions of neurons projecting ipsilaterally to area PMLS to those with callosal projections to the area 17/18 border (Fig. 4C). The distributions of the two neuron types in the area $17 / 18$ border region were similar. As described for Figure $4, A$ and $B$, the positions of ipsilateral projection neurons extended superficially in layer II/III up to the border belween layers I and II, whereas neurons with homotopic callosal projections were located in lower layer III as well as in upper layer IV. A single double-labeled neuron is indicated at the layer III/IV border of area 18 (asterisk in low-magnification section of Fig. 4C). One additional double-labeled neuron was found in area 17 of another section from this animal. These two double-labeled neurons were found within a total of 15 sections examined within the region of good overlap of Fast Blue and Diamidino Yellow labeling in the area $17 / 18$ border region.

Neurons with homotopic and heterotopic callosal projections in area PMLS. The section illustrated in Figure $5 \mathrm{~A}$ provides a clear demonstration of the differences in laminar distributions of callosal neurons projecting to area PMLS versus those projecting to the area $17 / 18$ border. Homotopic callosal neurons are located primarily in layer III, but also in layers $\mathrm{V}$ and $\mathrm{VI}$. Heterotopic callosal neurons projecting to the area $17 / 18$ border are located almost exclusively in layer VI. It is noteworthy that, in the deep layers, neurons with homotopic callosal projections are spread throughout both layers $V$ and $\mathrm{Vl}$, whereas heterotopic neurons are restricted to layer VI. This general pattern is also seen for neurons with heterotopic callosal projections in Figure $5 B$ and for neurons with homotopic callosal projections in Figure 5C. As was the case for areas 17 and 18, no double-labeled neurons were found in area PMLS when homotopic and heterotopic callosal projection neurons were labeled simultaneously.

Neurons with ipsilateral corticocortical and heterotopic callosal projections in area PMLS. Bilateral injections of the area $17 / 18$ border region (Fig. 5B) labeled neurons with heterotopic callosal and ipsilateral corticocortical projections in area PMLS. These two neuron types were intermixed and were most common in layers $V$ and VI. A single double-labeled neuron is illustrated in Figure $5 B$ (high and low magnification); in this experiment a total of three 
double-labeled neurons were found within 11 sections in area PMLS of the left hemisphere, and one double-labeled neuron was found in the right area PMLS. The other cat receiving bilateral area $17 / 18$ border injections had the highest number of double-labeled neurons in this study. Figure 6 contains a computer-microscope plot of the section with the highest frequency of double labeling from that experiment. All Diamidino Yellow-, Fast Blue-, and double-labeled neurons were counted in the region extending from the fundus to the medial edge of the middle suprasylvian sulcus. In total there were 422 Diamidino Yellow-labeled neurons, 53 Fast Blue-labeled neurons, and 8 double-labeled neurons. Thus, within the section illustrated, $1.9 \%$ of the neurons projecting ipsilaterally to the area $17 / 18$ border region also sent an axon collateral to the same cortical region in the opposite herrisphere, and $15.1 \%$ of the neurons projecting contralaterally also maintained an ipsilateral projection. As in every other case studied, there was no apparent tangential segregation of the two neuron types.

Neurons with ipsilateral corticocortical and homotopic callosal projections in area PMLS. Following injections of the ipsilateral area 17/18 border region and contralateral area PMLS (Fig. 5C), neurons in PMLS labeled by injections of the ipsilateral $17 / 18$ border region were found most frequently in layers $V$ and $V I$ but were also present in layers II, III, and IV. PMLS neurons labeled by the injection of contralateral PMLS were most prevalent in layer ill but were also present in layers $\mathrm{I}, \mathrm{V}$, and VI. Despite differences in their primary laminae of origin, the distributions of these two neuron types overlapped in layers III, V, and VI. No double-labeled neurons were found in the section illustrated. Only one double-labeled neuron was found in nine sections examincd within the region of good overlap of Fast Blue and Diamidino Yellow labeling. The two neuron types did not appear to be tangentially segregated

\section{Discussion}

The results of this study imply that most corticocortical neurons in the cat area $17 / 18$ border region and in area PMLS establish selective projections to a single cortical area. We made large multiple injections that produced regions of overlap between the two fluorescently labeled neuron populations that extended an average of 4200 $\mu \mathrm{m}$ (range of 1800 to $9600 \mu \mathrm{m}$ ) in the rostrocaudal dimension. Within these regions of overlap, the laminar distributions of neurons with different projections were often quite similar, and individual neurons with differing projections were frequently found immediately adjacent to one another. In every case, double-labeled neurons were rare, or even nonexistent. At the area $17 / 18$ border and in area PMLS no neurons could be double labeled by simultaneous injections of these two regions in the contralateral hemisphere in two cats. However, in both regions a few neurons could be double labeled by combined ipsilateral and contralateral injections. At the area $17 / 18$ border, a total of six double labcled ncurons were found in a total of three cats injected; five of these double-labeled neurons were in area 18. In area PMLS, the most common type of double-labeled neuron was one projecting bilaterally to the area $17 / 18$ border region. The highest frequency of double labeling found in a total of two cats examined with bilateral area $17 / 18$ border injections was 8 double-labeled neurons in a total of 475 labeled neurons $(1.7 \%)$ in a single section through area PMLS. In the single cat with injections of the ipsilateral area $17 / 18$ border region and contralateral area PMLS, only one double-labeled neuron was found in area PMLS.

Evidence suggesting a relative scarcity of branched corticocortical axons does not coincide with the pattern of axonal connections in other parts of the visual system. Branched axons appear to be much more common in the retinotugal, thalamocortical, and visual corticofugal systems (Bullier et al., 1984a; Kaufman et al., 1984; see reviews by Giolli and Towns, 1980, and Swadlow, 1983). In our richest case of double labeling (Fig. 6), only $15 \%$ of the neurons with contralateral projections also sent branches ipsilaterally. In contrast, studies of the visual corticofugal system indicate that $50 \%$ of corticopontine neurons send a branch to the superior colliculus, and $40 \%$ of corticotectal neurons send axon collaterals into the thalamus (Gibson et al., 1978; Swadlow and Weyand, 1981). However, several other studies are in agreement with our finding that most corticocortical neurons project to only one cortical area. Gilbert and Wiesel (1981), by using fluorescent tracers, found that neurons in area 17 projecting to areas 18 or 19 belonged to distinct populations. Schwartz and Goldman-Rakic (1982) found that $95 \%$ of the neurons labeled by fluorescent dyes in macaque cortex projected to only one of the three cortical termirnation sites they tested. Using antidromic activation in rabbit visual cortex, Swadlow and Weyand (1981) were unable to find corticocortical neurons with branched axons. A recent study with fluorescent tracers by Bullier et al. (1984b) indicates that, with a few exceptions, corticocortical neurons projecting ipsilaterally within cat visual cortex do not branch. In addition, these authors found that, among the several visual areas examined, areas 17 and 18 have the smallest number of ipsilaterally projecting corticocortical neurons with branched axons. Our comparison of ipsilaterally and contralaterally projecting neurons is in agreement with their findings. We found greater numbers of double-labeled neurons in area PMLS than in areas 17 or 18.

We have examined only three possible projections. The area 17/ 18 border region projects to about six contralateral visual areas and to at least six ipsilateral areas (Segraves and Rosenquist, 1982b; Symonds and Rosenquist, 1984a). Arca PMLS projects to nine contralateral visual areas and to at least seven ipsilateral ones. In addition, there are numerous corticofugal projections, including, for example, those to the dorsal LGN, the medial interlaminar nucleus the lateral posterior complex, and the superior colliculus (Gilbert and Kelly, 1975; Updyke, 1977, 1981). It is quite possible that the neurons that we have labeled send axon collaterals to one or more of these other termination sites. These possibilities remain to be investigated. However, Weber et al. (1983) have recently found that only 1 to $5 \%$ of Fast Blue-labeled corticotectal neurons in areas 17 and PMLS were labeled by Nuclear Yellow injections throughout the contralateral visual cortex.

In spite of the above limitations, cortical neurons appear to have a high degree of selectivity for other cortical targets. This selectivity seems to be acquired early in ontogenesis (Innocenti and Clarke, 1983). What could its function be? The selective projections could maintain a precise channeling of differential information to different cortical targets down to the single neuron level. On the other hand, there may in fact be a redundancy of processing among adjacent neurons with different termination sites. It will certainly require more intensive physiological studies to reveal processing differences that complement the anatomical differences. It should also be noted that the existence of extensive branching in corticocortical projections would not necessarily cause the same patterns of impulses to be sent to multiple cortical areas. Studies of impulse conduction in branched axons have shown that substantial differences can exist between the patterns of impulses conducted through branches of the same parent axon (Grossman et al., 1973; Swadlow et al., 1980; Swadlow, 1983).

Regarding the laminar distributions of the three corticocortical neuron types, our fluorescent tracer data are in agreement with existing HRP studies (for example, Gilbert and Kelly, 1975; Shatz, 1977; Keller and Innocenti, 1981; Segraves and Rosenquist, 1982a, b) and are summarized in Table I. In the area $17 / 18$ border region, neurons projecting ipsilaterally to area PMLS are primarily distributed throughout layer II/III with a few neurons in layers IV, V, and VI. Most of the homotopic and heterotopic callosal neurons in the area 17/ 18 border region are in the lower half of layer $\|/\| \|$ or in upper layer $N$, but a few are also found in other layers, most consistently in layer VI. Callosal neurons in layer VI of areas 17 and 18 had previously not been visualized by HRP injections into the suprasylvian region (Keller and Innocenti, 1981). In area PMLS, neurons projecting ipsilaterally to the area $17 / 18$ border region are located primarily in layers $V$ and $V I$ but are also found in smaller numbers throughout layers II and III, as well as in layer IV. Homotopic callosal neurons are 
located primarily in layer III but are also found in layers $\mathrm{H}, \mathrm{V}$, and VI. Heterotopic callosal neurons projecting to the area $17 / 18$ border region are distributed almost exclusively within layer $\mathrm{VI}$, a few are also present in layer III.

Because it enabled us to label two neuron populations within a single brain section, the double-tracer technique has unveiled subtle differences in the laminar distributions of callosal neurons which were not apparent in single-tracer HRP studies. First, our data suggest that in the superficial subzone of callosal neurons at the area $17 / 18$ border, homotopic callosal neurons are located in the lower half of layer I/III and in layer IV, whereas heterotopic callosal neurons projecting to area PMLS are restricted to the lower portion of layer II/III. Second, within the deep subzone of callosal neurons in PMLS, homotopic callosal neurons are located throughout layers $V$ and $\mathrm{VI}$, whereas heterotopic neurons projecting to the contralateral area $17 / 18$ border are restricted to layer VI. Qualitatively, these patterns of organization are quite convincing; however, verification of these findings will require a quantitative examination. In relation to these results, Gilbert and Kelly (1975) found that area 17 neurons projecting to ipsilateral area 19 were concentrated in the upper part of layer II/III, whereas neurons projecting to the ipsilateral area PMLS were located throughout the layer. Gilbert and Wiesel (1981) later reported that neurons in this same layer that project ipsilaterally to area 18 are located more deeply than those projecting to area 19. This finding has recently been confirmed by Bullier et al. (1984b). Taken in combination with our results, it is possible to compare the relative distribution of three ipsilaterally and two contralaterally projecting neuron types within layer II/III and upper layer IV of area 17. Cells projecting to ipsilateral area 19 are located predominantly in the upper portion of layer II/lll. Neurons projecting ipsilaterally to area PMLS are distributed throughout layer II/II. Neurons projecting to contralateral area PMLS are located within the lower half of layer II/ III. Finally, neurons projecting to ipsilateral area 18 as well as neurons projecting contralaterally to the area $17 / 18$ border region are located within the lower portion of layer $\|/\| l l$, and their distributions extend into the upper portion of layer IV.

We did not see any evidence for a tangential organization of ipsilaterally and contralaterally projecting neurons in either the area $17 / 18$ border region or in area PMLS. The two labeled neuron types were always intermixed, and they were roughly evenly distributed in a tangential plane; that is, there did not appear to be zones free of labeled neurons interspersed with zones of labeled neurons. This result is in agreement with that of Schwartz and Goldman-Rakic (1982), who found that fluorescent labeled callosal and ipsilaterally projecting neurons were always intermixed - not segregated in areas of the macaque's frontal, parietal, and cingulate cortex. Gilbert and Wiesel (1981) found that neurons in area 17 projecting ipsilaterally to areas 18 or 19 were roughly coextensive. Our results appear to conflict with previous studies which have found clusters of ipsilaterally projecting neurons in cat area 17 and in area PMLS (Gilbert and Wiesel, 1980, 1981: Bullier et al., 1984b; Symonds and Rosenquist, 1984a) and clusters of homotopic callosal neurons in areas 18 and PMLS (Lamont and Colonnier, 1978; Segraves and Rosenquist, 1982b). However, it is probable that the large multiple injections used in our experiments to ensure an extensive overlap of topography between injection areas were inappropriate for demonstrating the clustering of corticocortical neurons reported in previous studies. HRP injections of $0.5 \mu l$ are already large enough to obscure the banding pattern of ipsilaterally projecting neurons in cat area 17 (Gilbert and Wiesel, 1980). In the squirrel monkey (Tigges et al., 1981), small injections of HRP demonstrated a clustered pattern of ipsilaterally projecting neurons in area 17 , whereas large injections obscured this pattern. In the present study, large injections were used to ensure the highest possibility of labeling axon collaterals. An important next step will be to make much smaller paired injections to examine the organization and interrelationships of various corticocortical neuron types at a finer level.

\section{References}

Bentivoglio, M., H. G. J. M. Kuypers, C. E. Catsman-Berrevoets, H. Loewe, and $O$. Dann (1980) Two new fluorescent retrograde neuronal tracers which are transported over long distances. Neurosci. Lell. 18: 25-30.

Berkley, M. A., and J. M. Sprague (1979) Striate cortex and visual acuity functions in the cat. J. Comp. Neurol. 187: 679-702.

Berlucchi, G., J. M. Sprague, A. Antonini, and A. Simoni (1979) Learning and interhemispheric transfer of visual pattern discriminations following unilateral suprasylvian lesions in split-chiasm cats. Exp. Brain Res. 34: 551 574.

Bullier, J., H. Kennedy, and W. Salinger (1984a) Bifurcation of subcortical afferents to visual areas 17,18 and 19 in the cat cortex. J. Comp. Neurol. 228: 309-328.

Bullier, J., H. Kennedy, and W. Salinger (1984b) Branching and laminar origin of projections between visual cortical areas in the cat. J. Comp. Neurol 228: $329-341$

Camarda, R., and G. Rizzolatti (1976) Visual receptive fields in the lateral suprasylvian area (Clare-Bishop area) of the cat. Brain Res. 101: 427 443

Gibson, A., J. Baker, G. Mower, F. Robinson, and M. Glickstein (1978) Bifurcation of the corticopontine projection in the cat. Soc. Neurosci. Abstr. 4: 629.

Gilbert, C. D., and J. P. Kelly (1975) The projections of cells in different layers of the cat's visual cortex. J. Comp. Neurol. 163: 81-106.

Gilbert, C. D., and T. N. Wiesel (1980) Interleaving projection bands in corticocortical connections. Soc. Neurosci. Abstr. 6: 315

Gilbert, C. D., and T. N. Wiesel (1981) Projection bands in visual cortex. Soc. Neurosci. Abstr. 7: 356

Giolli, R. A., and L. C. Towns (1980) A review of axon collateralization in mammalian visual system. Brain Behav. Evol. 17: 364-390.

Grossman, Y., M. E. Spira, and I. Parnas (1973) Differential flow of information into branches of a single axon. Brain Res. 64: 379-386.

Innocenti, G. M. (1980) The primary visual pathway through the corpus callosum: Morphological and functional aspects in the cat. Arch. Ital. Biol. 118: 124-188.

Innocenti, G. M., and S. Clarke (1983) Multiple sets of visual cortical neurons projecting transitorily through the corpus callosum. Neurosci. Lett. 41:2732.

Kaufman, E. F. S., A. C. Rosenquist, and D. Raczkowski (1984) The projections of single thalamic neurons onto multiple visual cortical areas in the cat. Brain Res. 298: 171-174.

Keller, G., and G. M. Innocenti (1981) Callosal connections of suprasylvian visual areas in the cat. Neuroscience 6: 703-712.

Keizer, K., H. G. J. M. Kuypers, A. M. Huisman, and O. Dann (1983) Diamidino yellow dihydrochloride (DY. $2 \mathrm{HCl}$ ); a new fluorescent retrograde neuronal tracer, which migrates only very slowly out of the cell. Exp. Brain Res. 51: 179-191.

Kuypers, H. G. J. M., C. E. Catsman-Berrevoets, and R. E. Padt (1977) Retrograde axonal transport of fluorescent substances in the rat's forebrain. Neurosci. Lett. 6: 127-135.

Lamont, $\mathrm{P}$., and M. Colonnier (1978) Cortico-cortical projections to area 18 in the cat: An HRP study. Son. Neurosci Abstr. 4:76

LeVay, S., and C. D. Gilbert (1976) Laminar patterns of geniculocortical projection in the cat. Brain Res. 113: 1-19.

Otsuka, R., and R. Hassler (1962) Uber Aufbau und Gliederung der corticalen Sehesphare bei der Katze. Arch. Psychiatr. Nervenkr. 203: 213-234.

Palmer, L. A., A. C. Rosenquist, and R. J. Tusa (1978) The retinotopic organization of lateral suprasylvian visual areas in the cat. J. Comp. Neurol. 177: $237-256$

Poljak, S. (1926) Die Verbindungen der Area Striata (intrahemisphaerale, kommissurale, palliodienzephalische, palliotektale Fasern) bei der Katze und deren funktionelle Bedeutung. Z. Ges. Neurol. Psychiatr. 100: 545563.

Raczkowski, D., and A. C. Rosenquist (1980) Connections of the parvocellular C-laminae of the dorsal lateral geniculate nucleus with the visual cortex in the cat. Brain Res. 199: 447-451.

Raczkowski, D., and A. C. Rosenquist (1983) Connections of the multiple visual areas with the lateral posterior-pulvinar complex in the cat. J. Neurosci. 3: 1912-1942.

Rosenquist, A. C., S. B. Edwards, and L. A. Palmer (1974) An autoradiographic study of the projections of the dorsal lateral geniculate nucleus and the posterior nucleus in the cat. Brain Res. 80: 71-93.

Sanides, D., and J. Hoffmann (1969) Cyto- and myeloarchitecture of the visual cortex of the cat and of surrounding integration cortices. J. Hirnforsch. 11: 79-104.

Schwartz, M. L., and P. S. Goldman-Rakic (1982) Single cortical neurones 
have axon collaterals to ipsilateral and contralateral cortex in fetal and adult primates. Nature 299: 154-155.

Segraves, M. A., and G. M. Innocenti (1982) An examination of the projections of cat visual callosal neurons using double retrograde tracers. Neurosci. Lett. Suppl. 10: S442

Segraves, M. A., and A. C. Rosenquist (1982a) The distribution of the cells of origin of callosal projections in cat visual cortex. J. Neurosci. 2: 1079 1089 .

Segraves, M. A., and A. C. Rosenquist (1982b) The afferent and efferent callosal connections of retinotopically defined areas in cat cortex. J. Neurosci. 2: 1090-1107.

Shatz, C. J. (1977) Anatomy of interhemispheric connections in the visual system of Boston Siamesc and ordinary cats. J. Comp. Ncurol. 173: 497. 518.

Shoumura, K. (1973) Pathway from dorsal geniculate nucieus to visual cortex (visual radiation) in cats. Brain Res, 49: 277-290.

Spear, P. D., and T. P. Baumann (1975) Receptive field characteristics of single neurons in lateral suprasyivian visual area of the cat. J. Neurophysiol. 38: $1403-1420$

Spear, P. D., S. Milier, and L. Ohman (1983) Effects of lateral suprasylvian visual cortex lesions on visual localization, discrimination, and attention in cats. Behav. Brain Res. 10: 339-359.

Sprague, J. M., J. Levy, A. DiBerardino, and B. Berlucchi (1977) Visual cortical areas mediating form discrimination in the cat. .1. Comp. Neurnol. 172: $441-488$.

Swadlow, H. A. (1983) Efferent systems of primary visual cortex: A review of structure and function. Brain Res. Rev. 6: 1-24.
Swadlow, H. A., and T. G. Weyand (1981) Efferent systems of the rabbit visual cortex: Laminar distribution of the cells of origin, axonal conduction velocities, and identification of axonal branches. J. Comp. Neurol. 203. 799-822.

Swadlow, H. A., J. D. Kocis, and S. G. Waxman (1980) Modulation of impulse conduction along the axonal tree. Annu. Rev. Biophys. Bioeng. 9: 142179.

Symonds, L. L., and A. C. Rosenquist (1984a) Cortico-cortical connections among visual areas in the cat. J. Comp. Neurol. 229: 1-38.

Symonds, L. L., and A. C. Rosenquist (1984b) Laminar origins of visual cortico-cortical connections in the cat. J. Comp. Neurol. 229: 39-47.

Tigges, J., M. Tigges, S. Anschel, N. A. Cross, W. D. Letbetter, and R. L. McBride (1981) Areal and laminar distribution of neurons interconnecting the central visual cortical areas $17,18,19$, and MT in squirrel monkey (Saimiri). J. Comp. Neurol. 202: 539-560.

Tong, L., R. E. Kalil, and P. D. Spear (1982) Thalamic projections to visual areas of the middle suprasylvian sulcus in the cat. J. Comp. Neurol. 212: 103-117.

Updyke, B. V. (1977) Topographic organization of the projections from cortical areas 17,18 and 19 onto the thalamus, pretectum and superior colliculus in the cat. J. Comp. Neurol. 173: 81-122.

Updyke, B. V. (1981) Projections from visual areas of the middle suprasylvian sulcus onto the lateral posterior complex and adjacent thalamic nuclei in cat. I. Comp Neurnol $201 \cdot 477-506$

Weber, J. T., R. W. Rieck, and H. J. Gould, III (1983) Interhemispheric and subcortical collaterals of single cortical neurons in the adult cat. Brain Res. 276: $333-338$ 